Regular Paper

\title{
Methodology of Measuring Minute Remanence up to Submicron Tesla Level
}

\author{
T. MINAMITANI ${ }^{* 1}$, D. WAKAURA ${ }^{* 2}$, S. YAMADA ${ }^{* 1}$, H. SAKAI $^{* 2}$ and J. FUJII ${ }^{* 3}$
}

\begin{abstract}
The remanence remaining in a volcanic rock is one of important measurement data in geophysics field. The sample of a rock has the remnant magnetization up to $10^{-9} \mathrm{~T}$. Generally the remote magnetic fields measured by SQUID gradiometer presume the remanence of a rock as a sample. We propose a new methodology by measuring a magnetic field inside a cylindrical hole in the rotating sample. We could develop the simple measurement without shield system and high sensitivity sensor. The paper described the measurement system and the measured results of the rock. We could measure the remanence of a volcanic stone quarried from the volcanic rocks at Mt.Aso, Japan.
\end{abstract}

Keywords: remanence, magnetic sensor, through-hole, rotation, geomagnetism.

(Received: 24 July 2014, Revised: 14 April 2015)

\section{Introduction}

The measurement of remanence of magnetic samples with low-level permeability, such as a rock, is one of the environmental measuring techniques. As the amplitude of remanence in a rock is much less than that of geomagnetism, a magnetic shield is indispensable in order to remove geomagnetism at the measurement. For eliminating the dependence of the shape of a sample, the sensor is much remote from the measurement object, then the magnitude of magnetic field is decreased rapidly. Therefore, it is necessary to use a high-sensitive magnetometer with SQUID sensor on the conventional measuring method. A measuring equipment become large-scale and complex, then an in-site measurement is difficult $[1,2]$.

To pay attention to the simplicity of measurement especially, we proposed the measurement methodology where the magnetic fields in an air-hole of a testing sample presume an amplitude and direction of remanence. The magnetic fields inside a hole are uniform and the amplitude is high in relation to remanent magnetization. In order to remove the geomagnetism, the rotation of a sample changes remanence to alternating component. The methodology allows simplicity in the measurement of remanance. The equipment based on the methodology becomes portable on in-site measurement because high sensitive magnetic sensor can be used without magnetic shielding.

The paper discussed the basic principle of the

\footnotetext{
Correspondence: T. MINAMITANI, Institute of Nature and Environmental Engineering, Kanazawa University, Kakuma-Machi, Kanazawa, Ishikawa 920-1192, Japan email: heoco9999@yahoo.com.sg

${ }^{* 1}$ Kanazawa University ${ }^{* 2}$ Toyama University

${ }^{* 3}$ Fukui University
}

proposed system and the verification of accuracy. We measured the remanence of a volcanic rock and shows the advantage of measuring low-level remanece.

\section{Methodology of minute remanence}

\subsection{Detection procedure of remanence}

The outline of the measuring system is shown in Fig. 1. The tested sample ideally used as a cubic shape and has an through hole in the center. The measured sample rotates with a constant speed on the axis of a hole. The magnetic sensor is set on an axis and the sensing direction ( $x$-axis) is perpendicular to a hole. The measured sample rotates with a constant speed on the axis of a hole. Then the signal of remanence fixed on the sample is picked up as alternating component with the rotating frequency. On the other hand, geomagnetism is remained to be dc component. By processing the signal of the sensor by high-pass filter, the geomagnetism as a dc component can be cut. The amplitude and phase of the ac component presume the vector of remanence of a sample.

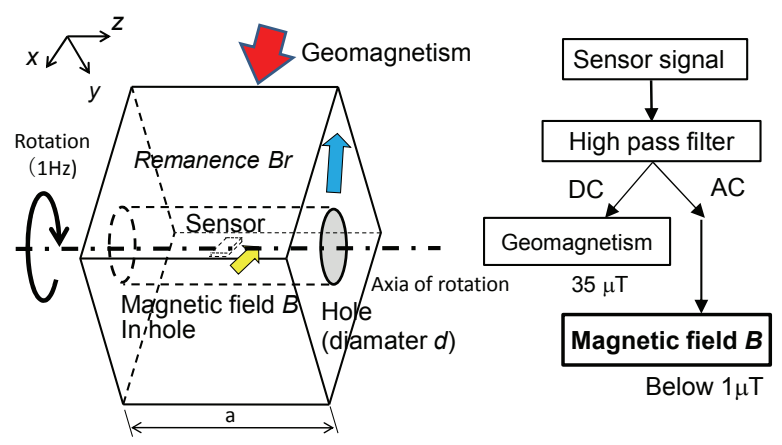

Fig. 1. Measurement system of remanence. 


\subsection{Relationship between a shape of sample and magnetic flux density in a hole}

We examine the relationship between the shape of measuring sample and the amplitude $B$ of magnetic flux density in a hole. The sample is cubic-shape with length $a$, and has a perpendicular air-hole with diameter $d$ as shown in Fig. 1. It is assumed that the remanence $B_{r}$ is magnetized uniformly. We simulated the ratio $\left(B / B_{r}\right)$ as a function of the shape ratio $(d / a)$ by numerical analysis. The ratio $\left(B / B_{r}\right)$ increases with decreasing $(d / a)$ gradually as shown in Fig. 2. The ratio $\left(B / B_{r}\right)$ by the shape ratio allows the estimation of remanence $B_{r}$ by measuring magnetic flux density $B$ inside a hole. The change of the ratio $\left(B / B_{r}\right)$ is only from 0.13 to 0.16 in the range of the ratio $(d / a), 0.15$ 0.45 .

Fig. 3 shows the magnetic flux distribution along the axis of hole $(z / a)$ when the ratio $(d / a)$ is 0.15 from 0.45 . It is clear that the magnetic flux density near the center of a hole is almost constant. This shows that the robustness over the position of a sensor is high inside a cylindrical hole.

\section{Measurement setup}

\subsection{Measurement equipment}

Fig. 4 shows the experimental equipment used in the experiment. The testing sample is rotated by a dc motor drive and the position of a sensor is adjusted on a

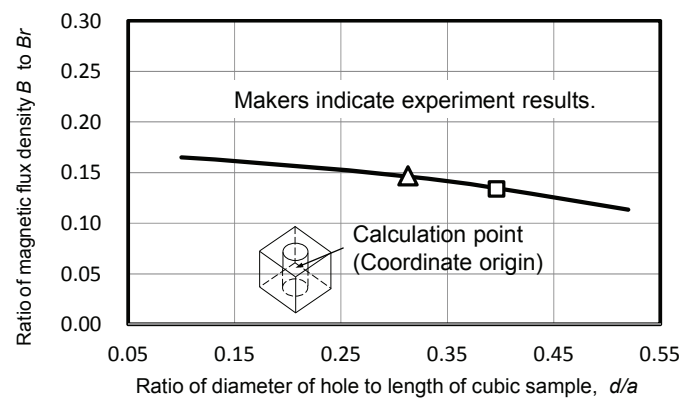

Fig. 2. Ratio of $B / B_{r}$ in the center of a hole.

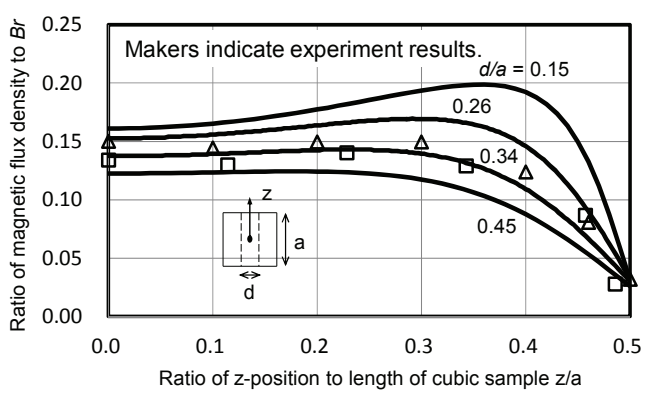

Fig. 3. Magnetic flux distribution along a hole- xyz stage. The distance between a motor and a sensor became large, then the magnetic noise by a motor is suppressed and the signal processing is applied. The rotational period of a motor is $1 \mathrm{~s}$.

Fig. 5 is a close-up picture of a sample. The sensor probe (Nano Tesla Sensor ${ }^{\odot}$ ) is based on a magnetic impedance sensor [4]. The size of probe is $11 \times 35 \mathrm{~mm}$ and $2 \mathrm{~mm}$ in thickness. The detecting range of magnetic change is up to $1 \mu \mathrm{T}$ and the noise is less than $1 \mathrm{nT}$ at $0.1-10^{4} \mathrm{~Hz}$. The dc signal is cut at the amplifier circuit. The head of the probe is inserted at the center of a hole.

\subsection{Testing sample}

We fabricated the testing samples with low susceptibility $\left(0<\chi^{*}<0.02\right)$ and low remanence $\left(B_{r}<1\right.$ $\mu \mathrm{T})$ instead of a rock included magnetite. The bulk material is composed of epoxy resin and magnetite powder. The weight density of magnetite is less than 0.1 $w t \%$. It has cubic structure with $35 \mathrm{~mm}$ in length. The

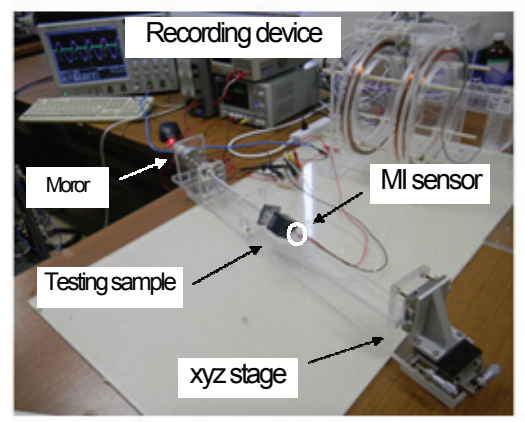

Fig. 4. Measuring system.

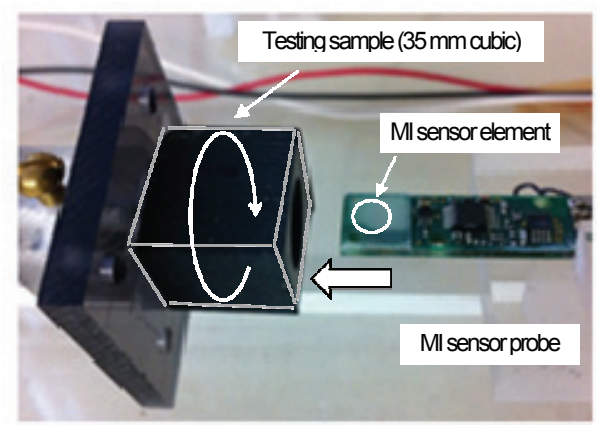

(a) Sample and sensor.

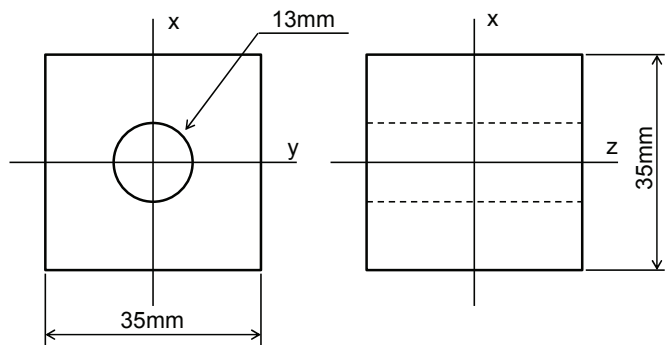

(b) Sample size.

Fig. 5. Configuration of magnetic sample and sensor. 
hole for the MI sensor is $13 \mathrm{~mm}$ in diameter. We examined the relationship between magnetizing field and remanence by vibrating magnetometer (VSM) as shown in Fig. 6. We used the Helmholz coils for magnetizing sample. We assumed that the remanence is uniformly in the sample. The hole is drilled after magnetization.

We fabricated three kinds of samples with different directions of remanence. The sample of type I has the only $\mathrm{x}$-axis remanence with different amplitudes. The sample is used for examining the relationship between remanence and magnetic flux density inside a hole. The sample of type II has the remanence with different magnetizing directions perpendicular to z-axis. The type II is used for examining the direction of remanence. The sample of type III has the remanence with $\mathrm{x}-\mathrm{z}$ plain perpendicular to y-axis. The type III is used for testing the influence of $\mathrm{z}$-component of remanence which can not be measured by the sensor.

\section{Measurement of remanence}

\subsection{Measurement by using test sample}

We examined the validity of the measurement methodology by using the above-mentioned samples. Fig. 7 shows the characteristics of magnetic flux density $B$ at the center of hole vs. the prescribed remanence $B_{r}$ by using the sample of type I. The magnetic field $B$ is proportional to the remanence $B_{r}$ by the gradient $k=$ 0.13 . The experimental value agreed with the simulated result by plotting the rectangular marker at $a / d=0.37$. The result denotes that the measured $B$ in a hole presumes the remanence $B_{r}$. Fig. 8 shows the waveform of direct output voltage of MI probe when the testing sample is rotated at $1 \mathrm{~Hz}$. We measured the fundamental component $B$ by using lock-in amplifier or Fourier expansion.

Fig. 9 shows the experimental results between the vector directions of the remanence and measured

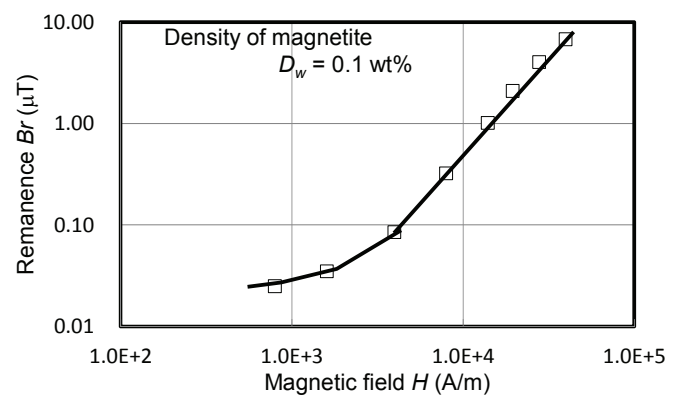

Fig. 6. Relationship between magnetizing field $H$ and remanence $B_{r}$. magnetic field by using the sample of type II. We recognized the good agreement of the direction. According to these results, we could estimate the moment (amplitude and direction) of remanence in the sample of low susceptibility.

Fig. 10 shows the experimental results between the remanence and magnetic fields by using the sample of type III. The type III sample has the magnetization with $x-z$ component, We measure the $x$-component of magnetic fields in a hole and estimate only the $x$ component of remanence $B_{r}$. The magnetic field $\mathrm{B}$ in a hole is proportional to $B_{r} \sin \theta$, then the magnetic field is parallel to the remanent vector. Fig. 11 shows the waveforms of the output voltage from the probe. These results denote that the $x$ and $y$ components of the magnetic field in a hole is independent of the $\mathrm{z}$ component of the remanence. We can conclude that the $x$ and $y$ components of the remanence can be estimated by measuring magnetic fields $\left(B_{x}\right.$ and $\left.B_{y}\right)$ in a hole along z-axis.

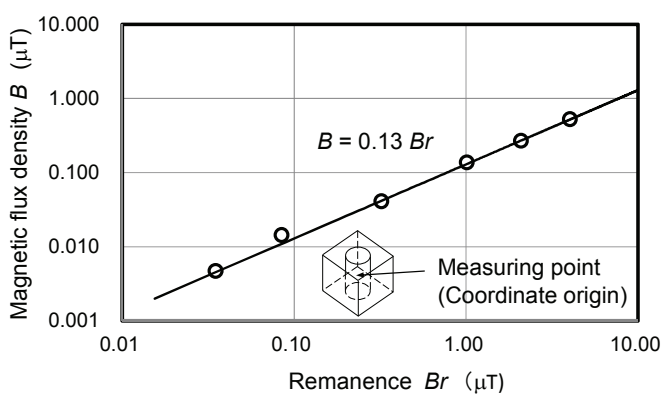

Fig. 7. Characteristics of magnetic field $B$ in a hole to remanence $B_{r}$.

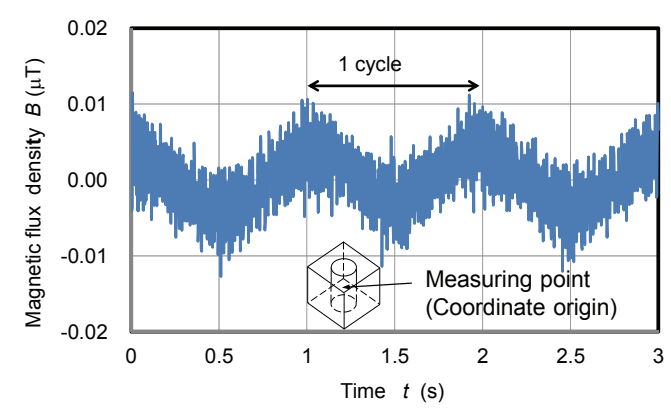

Fig. 8. Waveform of magnetic flux density in a

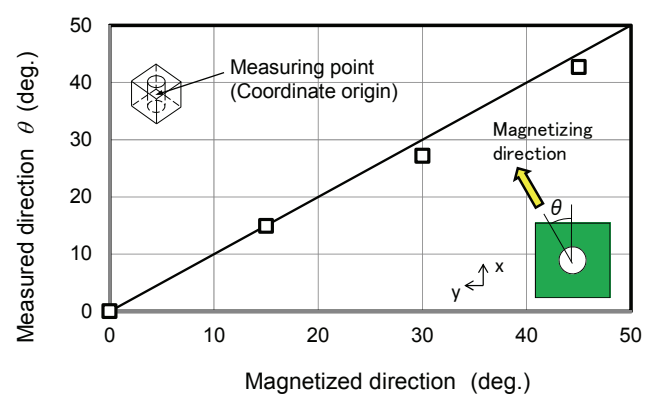

Fig. 9. Relation between magnetizing direction and flux density vector in a hole. 


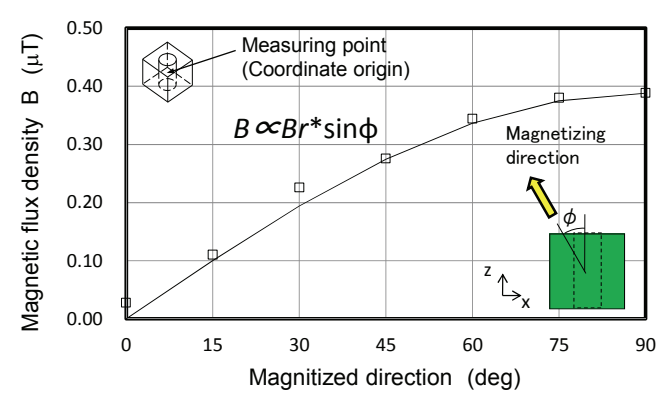

Fig. 10. Relationship between magnetizing direction and flux density vector in a hole.

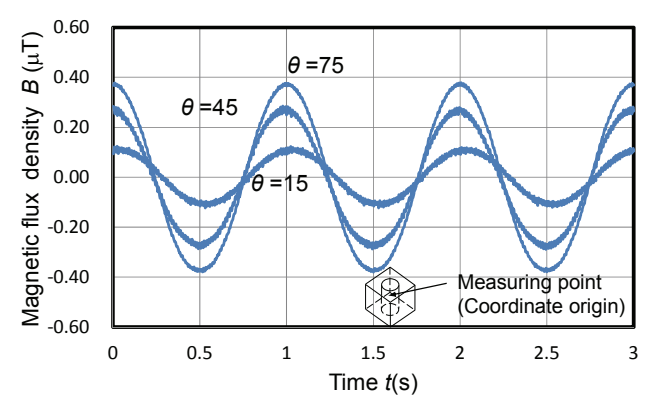

Fig. 11. Waveform of magnetic flux density in a hole.

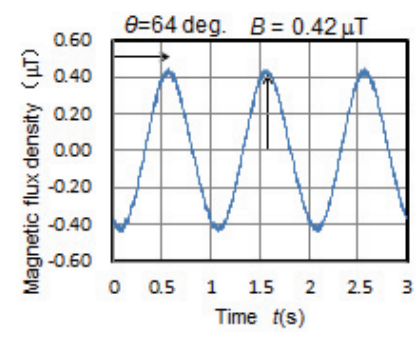

$a=35 \mathrm{~mm} \quad \mathrm{~d}=18 \mathrm{~mm}$ $\mathrm{d} / \mathrm{a}=0.51$

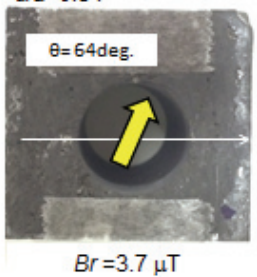

(a) Sample A
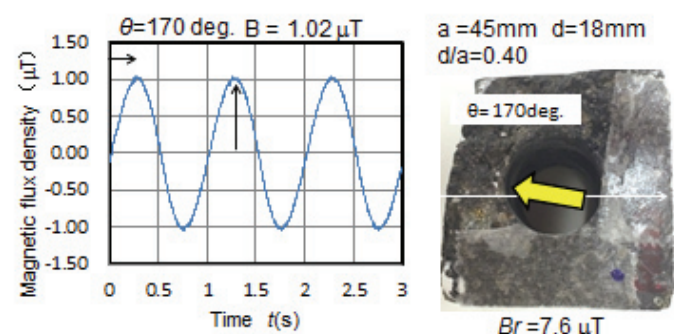

(b) Sample B.
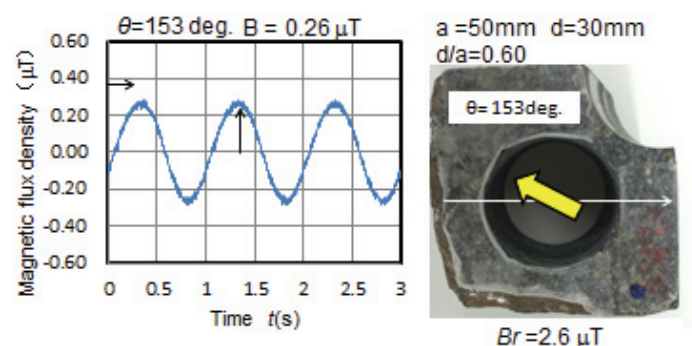

(c) Sample C.

\subsection{Measurement of remanence on volcanic rock}

We applied the system to measuring the remanence of volcanic rock. The three pieces of rocks are extracted at Mt.Aso, Japan. After spouting from the crater, a rock hardened under the geomagnetism. A testing sample is processed into the shape of the $35 \mathrm{~mm}$ cubic from the rock. But the shape is a little distorted structure with some cracks and unlike the model as shown in Fig.5. Fig. 12 shows the magnetizing direction of $x-y$ plain on the rock. The hole is used for measurement. The magnitude of the magnetic flux density in a hole is from 0.26 to $1.02 \mu \mathrm{T}$. The results prove that we can estimate the remanence of the volcanic rock.

\section{Conclusion}

We proposed the methodology and the measurement system of low-level remanence remained in a natural rock. We can estimate the $x$ - and $y$-axis components of remanence in a rock by measuring magnetic field inside the $z$-axis through hole of rock. The measurement for some volcanic rocks shows that the measurement becomes valid.

We need two holes in a rock to measure 3 components of remanence. In order to remove the interference of two holes, we drill two small holes orthogonally and suppress the effect of each others. We will apply the needle type giant magnetoresistance probe to the measurement [5].

\section{References}

[1] H.Kanaya, S.Okuma, "Physical properties of Cretaceous to Paleogene granitic rocks in Japan: Part 3. A case of the Ashio Belt (northern Kanto region)," Bull. Ggeol. Surv. Japan, 61, 1/2, pp.1-15, 2010.

[2] H.Sakai, S. Nagata, K.Masugata, "Propagation of IG Underground Elucidated by Remanent Magnetization," IEEJ Trans. on Power and Energy, Vol.133, No.9, pp.694-699, 2012.

[3] T.Minamitani, D.Wakaura, S.Yamada, "Research on Minute Remnant Magnetization Measurement of a Magnetic Body," Digest of 2014 JIEE Annual Conference, 2-127, 2014.

[4] Nanotesla sensor, Aichi Micro Intelligent Co. "Nano Tesla Sensor," http: //www.aichi-mi.com/magnetometer/ type-dh_en .htm.

[5] S.Kanamori, S.Yamada, T.Ueno, "2-D Image of EddyCurrent Testing and Dependence of Shape for Notch inside of Narrow Hole by Needle-Type Probe," Journal of Magnetics and Society of Japan, Vol.38, pp.21-24, 2014.

Fig. 12. Measurement of volcanic rocks. 